\title{
Jardin interdit au cœur de l'Éden. Conflits d'intérêts dans le Parc National du Manu (Pérou)
}

Jardín prohibido en el corazón del Edén. Conflictos de intereses en el Parque Nacional de Manu (Perú)

Forbidden garden in the heart of the Eden. Conflicts of interests in the Manu National Park (Peru)

\section{Fany Puygrenier Vargas}

\section{(Q) OpenEdition}

\section{Journals}

Edición electrónica

URL: http://journals.openedition.org/bifea/3707

DOI: $10.4000 /$ bifea.3707

ISSN: 2076-5827

\section{Editor}

Institut Français d'Études Andines

\section{Edición impresa}

Fecha de publicación: 1 diciembre 2007

Paginación: 407-426

ISSN: 0303-7495

\section{Referencia electrónica}

Fany Puygrenier Vargas, « Jardin interdit au cœur de l'Éden. Conflits d'intérêts dans le Parc National du Manu (Pérou) », Bulletin de l'Institut français d'études andines [En línea], 36 (3) | 2007, Publicado el 01 junio 2008, consultado el 04 diciembre 2020. URL : http://journals.openedition.org/bifea/3707 ; DOI : https://doi.org/10.4000/bifea.3707

\section{(c) $(1) \odot$}

Les contenus du Bulletin de l'Institut français d'études andines sont mis à disposition selon les termes de la licence Creative Commons Attribution - Pas d'Utilisation Commerciale - Pas de Modification 4.0 International. 


\title{
Jardin interdit au cœur de l'Éden Conflits d'intérêts dans le Parc National du Manu (Pérou)
}

\author{
Fany Puygrenier Vargas*
}

\begin{abstract}
Résumé
Conçues selon les seuls critères occidentaux pour préserver des écosystèmes mis en péril par la surexploitation des ressources, les aires naturelles protégées sont le théâtre d'enjeux idéologiques, politiques, économiques et sociaux. Instaurées sur des terres habitées, elles sont sources de conflits entre les autorités chargées d'en assurer l'intégrité et des populations locales ayant des pratiques et intérêts propres, divergents de ceux des conservationnistes. C'est par exemple le cas du parc national du Manu, créé en 1973 dans le Sud-est du Pérou. Dans le présent article, nous verrons comment l'administration de ce parc et les populations matsiguenga qui y résident, coexistent au sein de cet espace différemment perçu, vécu et ainsi culturellement construit.
\end{abstract}

Mots clés: Pérou, Manu, Parc National, conservation de la biodiversité, conflits d'intérêt, Matsiguenga, revendications territoriales

\section{Jardín prohibido en el corazón del Edén. Conflictos de intereses en el Parque Nacional de Manu (Perú)}

\section{Resumen}

Concebidas únicamente según criterios occidentales para preservar unos ecosistemas que peligran a causa de la explotación abusiva de los recursos naturales, las áreas naturales protegidas son el teatro de tensiones ideológicas, políticas, económicas y sociales. Establecidas en tierras pobladas, son fuente de conflictos entre las autoridades encargadas de asegurar su integridad y diversas poblaciones locales con prácticas e intereses propios, que divergen de los de los conservacionistas. Es por ejemplo el caso

* Doctorante en Anthropologie sociale et Ethnologie (EHESS, Paris), rattachée au Laboratoire d'Anthropologie sociale (Paris)E-mail : fanypuy@hotmail.com 
del Parque Nacional del Manu, creado en 1973 en el sureste del Perú. En el presente artículo, veremos cómo la administración de este parque y las poblaciones matsiguenga que allí viven, coexisten en el seno de este espacio que perciben, viven y así culturalmente construyen, de manera diferente.

Palabras clave: Perú, Manu, Parque Nacional, conservación de la biodiversidad, conflictos de intereses, Matsiguenga, reivindicaciones territoriales

\title{
Forbidden garden in the heart of the Eden. Conflicts of interests in the Manu National Park (Peru)
}

\begin{abstract}
Conceived in accordance with only Western criteria to preserve ecosystems endangered by the over exploitation of resources, protected natural zones are the theatre of ideological, political, economic and social stakes. Established on inhabited lands, they are the source of conflicts between the authorities, in charge of ensuring their integrity, and local population which have their own practises and interests, different from those of the conservationists. It is, for example, the case of the Manu National Park, created in 1973 in the south-east Peru. In this article, we will see how the administration of this park and the Matsiguenga populations who live in it, coexist within this area, which is perceived, lived and thus culturally constructed, in different ways.
\end{abstract}

Key words: Peru, Manu, National Park, biodiversity conservation, conflicts of interests, Matsiguenga, territorial claims

\section{INTRODUCTION}

Voici plusieurs mois déjà, lorsque les gardes du parc national du Manu s'aperçurent, au cours de l'une de leurs patrouilles, qu'un jardin avait été ouvert non loin de la Cocha Salvador, ils intervinrent immédiatement en le détruisant totalement. Certes les Matsiguenga de Tayakome et Yomybato avaient obtenu là une concession de 6 ha afin de construire, puis de gérer une auberge touristique dans la zone réservée à cet effet, mais ils n'étaient nullement autorisés à y mettre en culture une quelconque parcelle de forêt, aussi petite soit-elle, pas même pour subvenir à leur propre alimentation. Aussi anecdotique qu'il puisse paraître, le fait relaté ici n'en est pas moins révélateur des tensions existantes entre les populations matsiguenga et l'administration du parc national du Manu.

Ce n'est pas un hasard si, au Manu comme ailleurs, parcs nationaux et populations indigènes se retrouvent liés malgré eux. Les territoires de celles-ci correspondent souvent aux régions écologiquement privilégiées pour instaurer ceux-là. Pourtant loin de mettre à contribution ces populations qui ont su préserver, voire enrichir leur environnement, c'est sans les prendre en compte que sont élaborées les politiques de conservation. Aujourd'hui, bien que de nouveaux modes de conception et de gestion des aires naturelles protégées soient mis en œuvre pour limiter les retombées sociales de la conservation, de nombreux conflits subsistent.

Zone de productions extractive et agricole, parc national assurant la conservation de la biodiversité, territoires résidentiels - temporaires ou permanents - de populations autochtones et immigrantes, destination touristique... sont diverses destinations dont le bassin du Manu est actuellement le théâtre. Ce sont autant de modalités d'usage et de représentation qui entrent en concurrence dans cet espace et dont les divergences 
provoqent des tensions entre les différents usagers. Le présent article 1 a pour principal objectif d'en rendre compte, en analysant plus particulièrement l'interface entre les Matsiguenga du Manu et les promoteurs du parc national2. Par le terme «promoteurs », je me réfère à un ensemble hétérogène d'acteurs dont le dénominateur commun est d'œuvrer pour la protection et la gestion du parc national du Manu. S'il sera ici davantage question des administrateurs, bureaucrates ou techniciens, appartenant à I'Institut National des Ressources Naturelles (INRENA-Ministère de l'Agriculture), nous verrons que scientifiques, représentants d'Organisations Non Gouvernementales (ONG) ou agents de Coopération internationale, etc., jouèrent aussi un rôle déterminant sur la scène du Manu, y compris en ce qui concerne les politiques menées vis-à-vis des populations locales. Cette interface entre promoteurs du parc national du Manu et populations matsiguenga est représentative des conflits d'intérêts rencontrés dans cette région du sud-est du Pérou, et, de manière plus générale, là où sont instaurées des aires naturelles protégées sur le territoire de populations autochtones qui ne sont pas concertées a priori.

\section{PARADISIAQUE MANU ?}

«Eden vivant : les forêts cachées du Manu »; «Manu. En route pour le Paradis »; « Le Paradis amazonien du Pérou : Manu...»...

À en croire ces intitulés de film documentaire, d'un article de presse ou d'un livre de photographies destinés à un large public, le Manu serait un paradis. Enfer vert ou El Dorado, terres des Amazones ou, plus récemment, poumon de la planète, I'Amazonie en général est, depuis le début du XVle siècle, l'objet de représentations fort contradictoires dans l'imaginaire occidental. Le fait qu'elle soit de mieux en mieux connue ne semble rien lui enlever de son mystère tant il s'agit d'un univers d'une grande complexité. Dans le cas présent, c'est la luxuriance de ses forêts tropicales qui est mise en avant, dans son aspect le plus « paradisiaque ». Cette image, aujourd'hui amplement véhiculée par les agences de voyages, est devenue « vendeuse » et correspond aux attentes de la majorité des touristes. Ceux-ci, essentiellement originaires d'Amérique du Nord et d'Europe, citadins déconnectés du monde rural, perçoivent généralement la forêt amazonienne, de par son apparente abondance, tel «un paradis perdu» (et, pour le coup, un temps retrouvé, au cours de leur bref voyage). C'est d'autant plus vrai lorsqu'ils visitent une aire naturelle protégée établie en forêt tropicale telle que le parc national du Manu, « un paradis à protéger». L'administration du parc national elle-même use d'un registre similaire pour en assurer la

1 Les données ici présentées ont principalement été recueillies au cours de quatre missions de terrain d'une durée de deux à quatre mois chacune, alors que j'occupais un poste de Volontaire civile internationale en tant que jeune chercheuse au sein de l'Institut Français d'Études Andines, du 1 1'r septembre 2002 au 31 août 2004, détachée par le Ministère des Affaires étrangères. Auparavant, deux missions exploratoires, de trois mois chacune, avaient été réalisées en 2001 et 2002, grâce aux financements du Legs Lelong (Centre National de la Recherche scientifique), d'une aide au terrain attribuée par l'École des Hautes Études en Sciences Sociales et de la bourse « Aires culturelles » du Ministère de la Recherche et du Ministère de l'Éducation nationale. Que l'ensemble de ces institutions se voient ici nouvellement remerciées pour leur appui.

2 Je remercie l'Institut Français d'Études Andines de me permettre de reprendre ici de façon plus approfondie cette thématique des rapports entre promoteurs du parc national du Manu et populations matsiguenga, abordée succinctement au cours d'une intervention lors de la journée d'étude : « Le gouvernement de la Nature : Aménager Protéger, Conserver », organisée le 5 décembre 2005, par le Laboratoire d'Anthropologie Sociale (Paris).

3 Le présent article se fait l'écho de l'intervention «Paraiso para quem ? Populações Indigenas e o Parque Nacional do Manu (Peru) », présentée par Glenn Shepard Jr et Klaus Rummenhoeller, en juillet 2000, lors de la $22^{\mathrm{e}}$ réunion brésilienne d'anthropologie, forum de recherche sur le thème : "Conflits socio-environnementaux et unités de conservation ». Compte-rendu consultable sur le web à l'adresse :

http://ftp.unb.br/pub/UNB/dan/F.3-22RBA/sessao2/sheparderummenhoeller.rtf 
promotion. Lorsqu'elle écrit par exemple, dans le préambule de la brochure qu'elle édita à l'occasion de son trentième anniversaire :

«La valeur unique et inestimable du Parc National du Manu réside en l'immense richesse de la flore et de la faune, qui jusqu'à aujourd'hui demeure inaltérée par l'homme. »

«Inaltéré par l'homme » le Manu ? Tel un éden primordial (avant même la création d'Adam et Eve) ? Un paradis sans homme, exempt de péché originel, qui serait ici à entendre comme «péché de civilisation »... cette civilisation combien destructrice! Sans plus exagérer, on se demandera s'il y a aujourd'hui sur terre un seul endroit qui demeure inaltéré par I'homme. En admettant qu'il existe encore de tels lieux, le Manu ne fait pas partie de ceux-là. Si le bassin du Manu fut tout d'abord déclaré « réserve nationale » en 1968, puis officiellement reconnu « parc national » en 1973, c'était dans le but de protéger une flore et une faune d'une richesse incontestablement remarquable ${ }^{4}$, mais alors mises en péril par les madereros 5 , chasseurs de peaux et autres colons 6 . Certes les endroits qui furent les plus exploités sont aujourd'hui en grande partie régénérés et ils le sont peut-être d'autant mieux que, dans cette contrée difficile d'accès, les fronts pionniers sont restés circonscrits aux rives des principaux cours d'eau — le Madre de Dios, I'Alto Madre de Dios et le Manu — sans quasiment pénétrer à l'intérieur des terres, ni pendant le boom du caoutchouc, qui marqua la région entre 1890 et 1920, ni dans les années 1960, à l'arrivée de la nouvelle vague d'exploitants dont il est à présent question.

Lorsque le bassin du Manu fut proclamé «réserve nationale», chasse et coupe de bois furent interdites sur 1400000 ha. Les colons furent peu à peu expulsés de la zone désormais réservée et commencèrent à s'installer en périphérie, sur l'Alto Madre de Dios, ou en aval, sur le Madre de Dios. Puis, quand le parc national du Manu fut officiellement instauré par le Décret Suprême $N^{\circ}$ 0644-73-AG, le 29 mai 1973, l'article $2^{\text {nd }}$ de ce décret stipulait que la Direction Générale de la Réforme Agraire et de l'Aménagement Rural du Ministère de l'Agriculture devait « se charger de la délocalisation des occupants des terres du Parc National $\gg$. Et ce, parce que de manière générale, nature et activités humaines sont considérées par les conservationnistes comme intrinsèquement antagonistes et incompatibles. Ainsi la création d'aires naturelles protégées — dans l'intention de préserver une «Nature vierge » - implique fréquemment l'expulsion des populations locales hors des limites des zones définies. Si, dans le cas présent, c'était déjà en grande partie chose faite en ce qui concerne les populations immigrantes, qu'en fut-il alors des populations indigènes de la zone ? Furent-elles, elles aussi, bannies du Manu?

\section{CHASSÉS DE L'ÉDEN... (DE JUSTESSE !)}

Le parc national du Manu fut défini uniquement selon des critères écologiques. En observant la carte de ce parc (cf. fig. 1) il apparaît clairement que ses limites correspondent

4 Pour les scientifiques naturalistes aussi, le Manu s'avère être paradisiaque. Dès 1969, est installée la première station biologique de Cocha Cashu. Depuis, de nombreux chercheurs, spécialisés en biologie et écologie tropicales, viennent $s^{\prime} y$ former et y poursuivre leurs travaux, contribuant ainsi à la renommée internationale du Manu dans le domaine scientifique. Je ne rentrerai pas ici dans le détail des recensements réalisés aux alentours de la station biologique de Cocha Cashu, attestant de la diversité biologique de cette forêt ombrophile tropicale. J'en profite simplement pour signaler que ces inventaires sont, par ailleurs, utilisés par les agences touristiques, qui font de la faune et de la flore, les principales attractions du Manu.

5 Maderero est un terme espagnol désignant les extracteurs forestiers.

6 Le terme de «colons» (traduit de l'espagnol : colonos) se réfère aux populations originaires des Andes ou de la Côte qui sont venues peupler la forêt amazonienne afin d'en exploiter les ressources naturelles. Cette « colonisation » fut fréquemment promue par l'État péruvien afin de limiter l'exode rural.

7 Extrait du Décret Suprême № 0644-73-AG (INRENA-SPMA, 2002 : 197). 


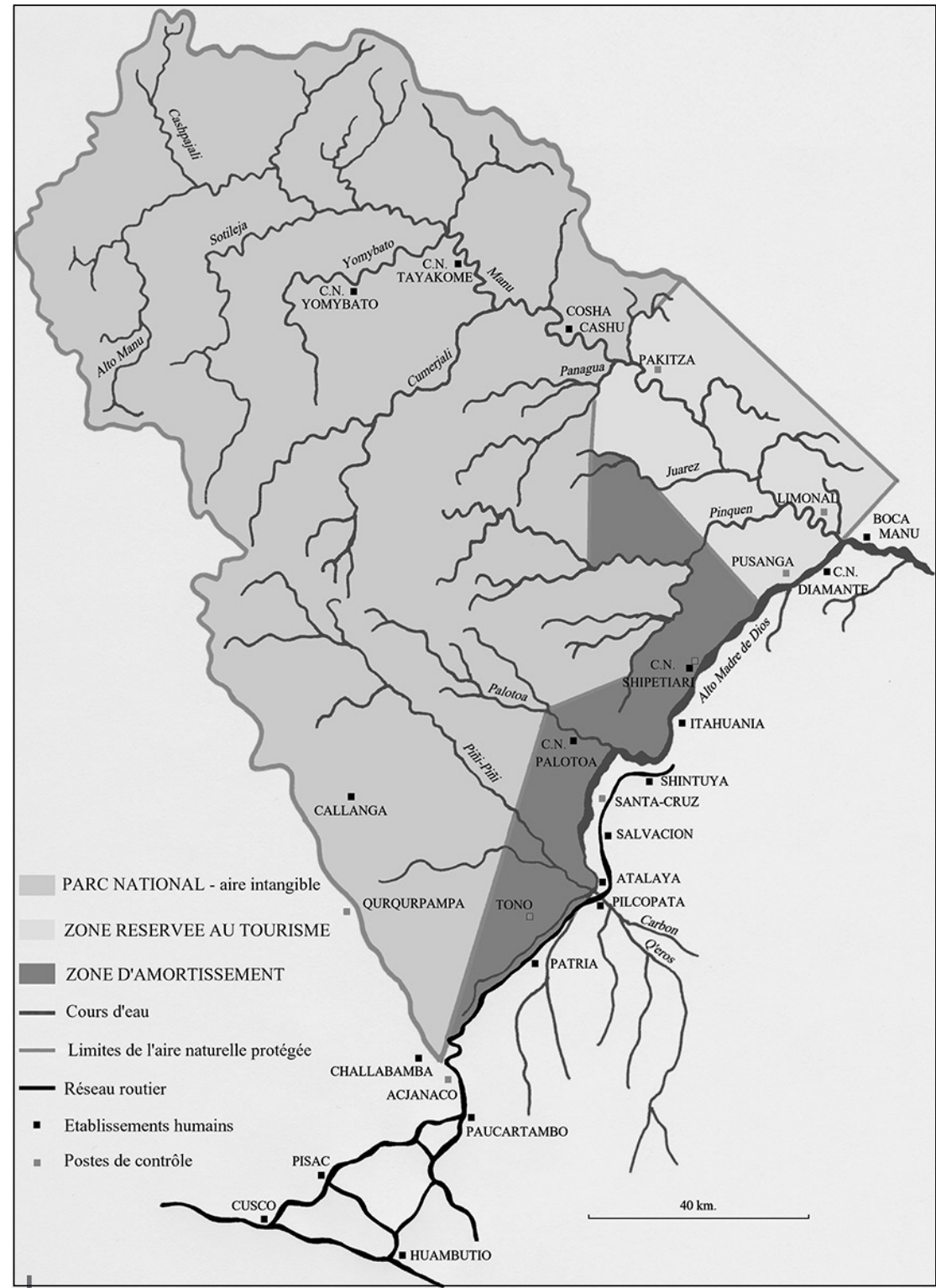

Fig. 1 - Parc National. Réserve de la biosphère du Manu

au pourtour des bassins du Manu et de ses affluents, ainsi que des bassins des affluents de la rive gauche de l'Alto Madre de Dios dont les principaux sont le Piñi-Piñi et le Palotoa. Cette délimitation ne prend pas en considération les territoires des populations indigènes vivant dans la région, qui furent morcelés par les frontières du parc national. Celui-ci fut instauré sans consultation préalable de ces populations. Pourtant le bassin du Manu est une région où se rencontrent les territoires de différents groupes ethniques appartenant aux 
familles linguistiques arawak, haramkbut et pano. II s'agit principalement de populations matsiguenga, yine (piro), amarakaeri, huachipaeri, yora (nahua) ainsi que d'autres groupes «non-contactés». En accord avec un vécu particulier vis-à-vis de la civilisation colonisatrice, les populations indigènes vivant dans le Manu ont adopté diverses formes de réponses sociales dans leurs rapports avec l'Étranger, allant de l'isolement « volontaire »8 à l'intégration progressive à l'économie de marché. De fait, les populations indigènes vivant isolées, dans des zones difficilement accessibles, ne furent pas inquiétées par la création du parc national du Manu. De plus, elles représentaient alors une moindre menace en comparaison des madereros qui avaient installé leurs scieries sur le bas Manu.

Parmi les populations indigènes du Manu, ce sont surtout les Matsiguenga installés à Tayakome qui causèrent des problèmes aux promoteurs du parc national. En 1971, le village de Tayakome comptait 209 personnes (d'Ans, 1981 : 155). Depuis une dizaine d'années, les missionnaires évangélistes de l'Institut Linguistique d'Été avaient rassemblé là, sur la rive droite du Manu, des familles matsiguenga originaires des cours supérieurs du Sotileja et du Cumerjali, deux affluents du Manu. Ils contactèrent ces familles qui vivaient de façon très dispersée par l'intermédiaire de guide-interprètes matsiguenga, natifs de l'Alto-Urubamba. Si le principal dessein de l'Institut Linguistique d'Été était de christianiser ces populations, il les incita aussi à vivre regroupées en village, instaura une école dont le professeur lui-même matsiguenga les instruisit dans leur langue, mit en place une assistance médicale et, surtout, commença par leur offrir des biens manufacturés fort valorisés, tels que des outils de métal, des habits, des ustensiles de cuisine, etc., qui les convainquirent peu à peu d'emménager dans ce centre de richesses. Rapidement, I'Institut Linguistique d'Été établit aussi un système de troc pour permettre aux Matsiguenga d'acquérir davantage de biens (en particulier des fusils et des munitions nécessaires à la chasse) en échange de peaux d'animaux.

Pour les autorités de la réserve nationale, ce trafic devait prendre fin. Il était impensable que ces populations continuent de chasser pour les besoins du troc des espèces dorénavant considérées comme «protégées», «menacées» voire « en voie d'extinction ». Afin que cessent ces activités, devenues illégales depuis la création de la réserve, la piste où I'avionnette atterrissait pour faciliter ce négoce fut fermée, les fusils confisqués. Pour surveiller les habitants de Tayakome afin qu'ils respectent les nouvelles règles qui leur étaient imposées, un poste de contrôle fut installé durant plusieurs années à proximité du village. Contrarié dans ses manœuvres, l'Institut Linguistique d'Été fut alors contraint de migrer à l'extérieur de la réserve et persuada la moitié de la population de Tayakome de le suivre pour créer le village de Segakiato, sur le bas Camisea (d'Ans, 1981).

De 1969 à 1971, André-Marcel d'Ans — alors expert mandaté par le gouvernement belge dans le but d'organiser un groupe d'enseignement et de recherche sur les langues amazoniennes à l'Université Nationale de San Marcos à Lima (et simultanément regagner le terrain conquis par I'Institut Linguistique d'Été en Amazonie péruvienne) - , avait par ailleurs été chargé par le Ministère de l'Agriculture d'évaluer et de mettre en place le programme anthropologique du parc national du Manu (d'Ans, 1981 : 162). En réaction au projet d'expulser manu militari les populations indigènes de la zone - comme il

8 Durant le boom du caoutchouc (fin XIXe-début XXe), pour échapper aux funestes razzias esclavagistes alors menées par les exploitants et leurs alliés dans le but de se procurer de la main d'œuvre, de nombreux Indigènes trouvèrent refuge aux sources des affluents des principaux fleuves de la région, dans des vallées encaissées dont leurs descendants défendent aujourd'hui encore l'entrée aux visiteurs étrangers. D'autres adoptèrent un mode de vie nomade. Depuis lors, de nombreux groupes indigènes vivent en isolement que I'on qualifie aujourd'hui de « volontaire », bien qu'il relève davantage d'une contrainte imposée par ces sombres événements que d'un choix à proprement parlé. 
avait été décidé, en partie, en suivant les conseils d'un expert de la FAO9 participant à la création du parc national - , ce « programme anthropologique » consistait alors à mettre en place des solutions qui permettraient aux Matsiguenga de conserver leur territoire dans les meilleures conditions possibles (André-Marcel d'Ans, communication personnelle). Notons que ce souci «anthropologique» émanant du Ministère de I'Agriculture de l'époque, du moins cette préoccupation pour le devenir de ces populations, dans la phase antérieure à l'instauration officielle du parc national, disparut ensuite des directives politiques de l'administration pendant des années. Il fallut, en effet, attendre 1985 pour que l'administration du parc national intègre dans son équipe un anthropologue et crée un premier "département d'anthropologie», département qui fut dissous trois ans à peine après sa création (Rummenhoeller \& Helberg Chavez, 1992 : 257-259). Par la suite, et jusqu'à aujourd'hui, I'administration du parc national du Manu engagea de façon intermitante des anthropologues pour de très courtes périodes. Cette conduite témoigne du peu de cas fait des populations indigènes du Manu, même si, paradoxalement, elles sont toujours considérées comme une véritable menace par les conservationnistes, chargés de la protection de cette aire naturelle protégée.

Parmi les alternatives proposées au début des années 1970, il avait été question, dans un premier temps, de réserver sur le bas Manu (en aval du confluent avec le Panagua) un «territoire de dégagement» qui aurait été «attribuable aux Matsiguenga après une période transitoire de développement à mener à Tayakome même, de telle sorte que puisse s'achever la concentration de tous les Matsiguenga du parc » (d'Ans, 1970 : 96). Il n'en fut rien. Quelques années plus tard, la dite zone fut bien déclarée «zone réservée » mais « à usage touristique ». Par ailleurs, les habitants de Tayakome qui avaient refusé de suivre I'Institut Linguistique d'Eté à destination de Segakiato, ne consentirent pas non plus à quitter leurs terres du Manu. Cependant, à la fin des années 1970, ils se dispersèrent : quelques familles restèrent aux environs de Tayakome, d'autres partirent s'installer en amont sur la Quebrada Fierro ou Yomybato, tandis que d'autres allèrent s'établir sur l'Alto Madre de Dios.

À défaut de n'avoir pu exclure les populations indigènes du Manu, l'administration les toléra finalement à l'intérieur du parc national, mais à la condition qu'elles

« [continuent] leurs pratiques et usages traditionnels pour satisfaire leurs besoins de subsistance, dans la mesure où ceux-ci sont compatibles avec les objectifs de l'aire [naturelle protégée] $\gg 10$.

Une telle approche, qualifiée à juste titre de "primitivisme renforcé » par Marcus Colchester (1999: 164), est caractéristique des politiques de conservation telles qu'elles ont été menées au cours du XXe siècle. En effet, pour les conservationnistes, la présence de populations indigènes au sein d'une aire naturelle protégée devient acceptable, si ces populations

«se conforment strictement aux stéréotypes que l'on a sur [elles] et qu'[elles] n'adoptent aucune pratique moderne » (Colchester, 1999 : 164).

Considérés alors comme de «Bons sauvages», les Indigènes sont assimilés à leur milieu naturel. De cette façon, ce dernier continue d'être tenu pour une «Nature vierge»,

9 La Food and Agriculture Organization (FAO) ou « organisation des Nations Unies pour l'alimentation et l'agriculture », est une institution qui a été créée en 1945 par l'ONU en vue de mieux répartir les produits agricoles et de lutter contre la faim dans le monde.

10 Extrait du Décret Suprême N 045-2002-AG qui établit l'extension de la superficie du Parc National du Manu (INRENA-SPMA, 2002 : 198-199). 
« inaltérée », malgré leur présence. La contradiction est ainsi levée. Mais, au même titre que cette Nature protégée par un parc, les Indigènes qui y résident, devraient eux aussi perdurer dans leur état « primitif », « sauvage».

$C^{\prime}$ est en ce sens que fut établi le règlement interne du parc national du Manu. Les Matsiguenga qui y résident, n'ont, par exemple, pas le droit d'acquérir de nouvelles technologies jugées nuisibles à leur environnement ou encore d'exploiter les ressources naturelles du parc à des fins commerciales. Néanmoins, il me faut souligner ici qu'il est davantage question de limiter les impacts négatifs qu'ils pourraient causer à leur environnement et, par conséquent, au parc national du Manu que d'interdire en soi leur développement technique et socio-économique. Par ailleurs, malgré les directives énoncées, la législation en faveur de la conservation et celle en faveur des peuples indigènes étant en totale contradiction, l'administration du parc national du Manu dut faire au fil des ans quelques concessions au profit des populations de Tayakome et Yomybato, dont tout me pousse à croire qu'aux yeux des conservationnistes, il s'agissait chaque fois d'autoriser le moindre pour éviter le pire.

\section{VERS UNE RECONNAISSANCE}

Ces dernières décennies, avec l'appui de différents acteurs, les Matsiguenga résidant à Tayakome et Yomybato, obtinrent parfois gain de cause pour « aller de l'avant » selon leur propre volonté et malgré les restrictions édictées par l'administration du parc. Je ne retiendrai ici que les étapes décisives du développement de Tayakome et Yomybato, souvent relatées par leurs habitants eux-mêmes. Par " développement», je ne me réfère pas seulement à une potentielle croissance économique (potentielle car elle reste très limitée) mais aussi, et surtout, à l'amélioration qualitative des conditions de vie de ces populations, en particulier dans les domaines matériel et sociopolitique.

Mis sur les rails de la «civilisation» par les missionnaires de l'Institut Linguistique d'Été une dizaine d'années avant la création du parc, les Matsiguenga originaires du Manu ne comprirent pas pourquoi ils durent renoncer à leurs nouveaux acquis à partir de 1973, lorsque le parc national du Manu fut officiellement créé. Finies l'instruction et I'assistance médicale, finis les fusils et autres biens fort utiles. Ils furent particulièrement décontenancés de ne plus recevoir aucune aide sanitaire alors qu'ils en auraient eu grand besoin lorsque se propagèrent des maladies respiratoires graves que seule la médecine occidentale était à même de soigner. L'Institut Linguistique d'Été parti, puis le premier programme anthropologique ayant périclité, les Matsiguenga de Tayakome et Yomybato furent, en quelque sorte, de nouveau livrés à eux-mêmes. Cependant, dans le but d'obtenir de menus objets, quelques hommes, jeunes et moins jeunes, partirent à l'extérieur offrir périodiquement leur force de travail. Une famille se rapprocha de la station biologique de Cocha Cashu pour faire du troc avec les scientifiques qui y menaient leurs recherches. Mais, de manière générale, l'administration du parc national fit en sorte de limiter les contacts que les habitants de Tayakome et Yomybato pouvaient entretenir avec l'extérieur, convaincue que le meilleur moyen pour qu'ils recouvrent un mode de vie «traditionnel» était de les maintenir isolés.

Toutefois, au début des années 1980, les missionnaires catholiques de la mission de Shintuya (Alto Madre de Dios) vinrent au secours de ces « âmes perdues », en commençant par apporter leur soutien à ces populations, tant sur le plan matériel que sur les plans sanitaire et éducatif. En 1982 à Tayakome, puis en 1984 à Yomybato, furent installées des écoles dirigées par des professeurs matsiguenga, originaires d'Urubamba. La constitution péruvienne garantissant à tous ses citoyens l'accès à l'éducation, l'administration ne put 
s'opposer à l'installation de ces deux écoles à l'intérieur du parc national. Appartenant à la RESSOP11, ces écoles sont administrées par les dominicains du vicariat apostolique du Madre de Dios, en vertu d'un contrat passé avec le Ministère de l'Éducation.

Depuis leur nomination, les professeurs, qui sont davantage au fait des démarches administratives et des normes légales, se sont engagés auprès des habitants de Tayakome et Yomybato afin de revendiquer la reconnaissance de leurs droits. Leurs actions sont d'autant mieux menées que les professeurs nommés à Yomybato appartiennent au Centre pour le Développement de I'Indigène Amazonien (CEDIA), une organisation non gouvernementale consacrée à la défense des droits indigènes. Ainsi, quelques années après leur affectation, le 22 janvier 1988, Tayakome et Yomybato furent reconnues « Communautés Natives». À ce titre, elles obtinrent une reconnaissance légale et devinrent des « personnes juridiques». Cependant, étant localisées à l'intérieur du parc national du Manu, aucune terre ne leur fut attribuée. Seul un droit d'usage leur est depuis concédé, dans la mesure où, nous l'avons vu précédemment, leurs activités restent compatibles avec les objectifs du parc.

Des années durant, les habitants de Tayakome et Yomybato, soutenus dans leur démarche par le CEDIA, puis par la Fédération Native du Madre de Dios (FENAMAD), revendiquèrent l'attribution de titres de propriété sur leurs terres pour avoir le droit d'en disposer comme bon leur semble. Sans succès... L'administration du parc national du Manu et ses promoteurs s'y opposèrent catégoriquement, considérant déjà comme un danger la présence de ces deux communautés natives en son sein. Au cours de mes séjours à Tayakome et Yomybato, j'ai constaté que l'attribution de titres de propriété sur les terres demeure un thème central, débattu fréquemment lors des assemblées extra-communales (réunissant les habitants de l'une ou l'autre de ces communautés et leurs visiteurs occasionnels). En revanche, la position des habitants de Tayakome et Yomybato a changé. Si, pour des raisons davantage politiques, les fédérations indigènes régionales poursuivent leurs actions pour que Tayakome et Yomybato obtiennent les titres de propriété de leurs terres, leurs habitants se sont ravisés. L'administration du parc national a su les convaincre qu'il leur est plus profitable de bénéficier de tout l'espace souhaité à l'intérieur du parc — puisqu'ils y ont une entière liberté de mouvement - plutôt que de se retrouver confinés sur la superficie exiguë qui leur appartiendrait en cas d'attribution de titres de propriété. L'argument semble pertinent au regard de leurs activités socio-économiques « traditionnelles».

\section{JARDINS MATSIGUENGA, UNE PRATIQUE DU TERRITOIRE}

Comme la majorité des peuples indigènes de la forêt amazonienne, les Matsiguenga pratiquent chasse, pêche et horticulture. Leurs modes d'exploitation des ressources naturelles exigent une forte mobilité. Chaque année, une famille matsiguenga ouvre un nouvel essart qui lui fournira en proportion l'essentiel de son alimentation. Lorsque les terres situées à proximité de sa maison, ne sont plus propices à leur mise en culture, elle part, à plus ou moins longue distance, s'installer sur des terres fertiles pour y défricher et planter un nouveau jardin. Elle peut parfois retourner sur des terres anciennement exploitées qui se sont depuis régénérées, après avoir été abandonnées durant plusieurs années. Si divers critères entrent en compte pour établir une nouvelle résidence, qu'ils soient «techniques» (qualité des sols, proximité d'un point d'eau, etc.) ou sociaux (être « à bonne distance » de ses proches, parents ou amis, c'est-à-dire ni trop près, pour préserver son intimité, ni trop loin, pour favoriser les échanges), chaque famille n'en est pas moins libre de s'installer où elle le souhaite.

11 RESSOP est le sigle espagnol de Red Escolar de la Selva del Sur-Oriente Peruano qui signifie en français « Réseau Scolaire de la Forêt du Sud-Est péruvien ». 
Pour les Matsiguenga, il n'existe pas de propriété foncière au sens où nous l'entendons. Toutefois les groupes et sous-groupes ont un territoire précis; chacun se reconnaissant de tel ou tel endroit. Sont identifiés et s'identifient par exemple comme tels les "gens de Cumerjali », les «gens de Soliteja », etc. Les cours d'eau et, plus récemment, les communautés natives constituent les principaux axes et points de référence des lieux de naissance ou de résidence de chacun. Précisons que le territoire est ici entendu comme une région qui inclut des habitations, des terres - qui peuvent être, sont ou ont été mises en culture - , des forêts de chasse et de collecte et des cours d'eau de pêche. Selon les contextes d'interlocution, un individu peut revendiquer son appartenance à des territoires de différentes échelles, dépendamment du fait qu'il se réfère aux espaces occupés par sa famille restreinte ou étendue, ou encore par le groupe résidentiel dont il fait partie et qui inclut toutes les familles élargies établies sur un même cours d'eau ou, à présent, au sein d'une communauté native, voire dans une plus vaste région. La cohésion entre ces différents groupes résidentiels est renforcée par l'exogamie. De la sorte se dessinent plusieurs séries de cercles territoriaux concentriques, enchevêtrés les uns aux autres et dont l'ensemble forme l'actuel territoire matsiguenga. Celui-ci s'étend aujourd'hui depuis le bassin de l'Urubamba à l'ouest jusqu'aux bassins de la rive droite du Manu et de la rive gauche de l'Alto Madre de Dios à l'est.

En raison de leur semi-nomadisme, les groupes locaux ne revendiquent aucune exclusivité sur des terres qu'ils n'occupent que temporairement. Les familles vont et viennent, se dispersent ou se (re)composent au fil des années. Chez les Matsiguenga, où les règles de résidence sont matrilocales, une jeune femme restera auprès de ses parents avec son mari, alors qu'un jeune homme partira plus ou moins loin du foyer parental pour trouver son épouse et s'intégrer à une nouvelle unité socio-économique. Avant obtenir le droit de prendre femme, le futur gendre est mis à l'essai : il doit montrer ses capacités à répondre aux besoins de sa famille en chassant mais surtout en faisant ses preuves au champ. En effet, les Matsiguenga accordent une importance particulière à l'horticulture. Si celle-ci est considérée comme l'activité féminine par excellence par la majorité des peuples amazoniens qui la pratiquent en sus de la chasse et de la pêche, elle est, pour les Matsiguenga, un travail principalement masculin. Alors que les autres hommes se contentent de défricher l'abattis, les hommes matsiguenga s'occupent aussi de planter et entretenir les essarts. Ils participent même parfois à la récolte et au portage de certaines denrées — bien que ces dernières tâches soient plus couramment dévolues aux femmes. D'après France-Marie Renard-Casevitz, il existe ainsi une complémentarité entre des femmes détentrices de terres et des hommes garants de techniques agricoles assurant leur exploitation. Par le mariage, les hommes accèdent à un territoire qui appartient aux femmes (Renard-Casevitz, 1972 : 228-231). Certes de forts liens de dépendance unissent les jeunes mariés aux parents de l'épouse et les obligent à rester dans les proches environs, mais peut-on dire pour autant que les terres appartiennent aux femmes, qu'elles en sont «maîtres » (Renard-Casevitz, 1972 : 229) ? II semble davantage que nul ne possède des terres en propre et tous peuvent potentiellement exercer l'usufruit de n'importe quelle terre. Comme France-Marie Renard-Casevitz l'écrit elle-même :

«Du fait de la rotation des jardins, on ne possède pas un champ fixe et individuel : ce qu'on détient plutôt c'est l'usufruit familial de parcelles déplacées au sein d'un territoire détenu collectivement et la propriété, stricto sensu, s'applique aux produits de ces parcelles — et de son travail —, non à la terre défrichée » (Renard-Casevitz, 1972 : 229).

Encore que défricher un terrain constitue un premier acte d'appropriation. Cette appropriation se marque dans l'espace, mais surtout dans le temps. Car une parcelle, une fois défrichée, appartient déjà à celui qui a commencé ainsi à la travailler, et elle sera 
sienne le temps de la préparer, de l'ensemencer, de la cultiver, de la récolter, puis de l'abandonner. En friche, elle ne lui appartiendra plus. Quelques années ou décennies plus tard, quiconque souhaitera utiliser cet espace redevenu productif le pourra, pour le temps d'un nouveau cycle seulement.

Les Matsiguenga considèrent donc la terre comme un bien collectif et surtout inaliénable. Aujourd'hui, ils contestent la division des terres en parcelles privées comme le préconisent les colons et préfèrent revendiquer la reconnaissance de terres accessibles à tous natifs pour continuer à en exploiter les ressources comme ils le désirent. Néanmoins, devant faire face à la colonisation de leurs territoires et afin de défendre leurs droits, les populations matsiguenga — de même que les autres peuples amazoniens résidant au Pérou —, ont dû généralement se plier aux instruments juridiques que la Constitution péruvienne mettait à leur disposition. La loi des communautés natives de 1974, décrétée par le gouvernement militaire du Général Velasco, fut un premier pas vers leur reconnaissance. Depuis, malgré la promulgation de nouvelles lois, les droits d'accès à la terre pour les populations indigènes restent réduits au minimum. À l'exception des rares « réserves communales » indigènes qui ont été instaurées (et dont les limites ne sont d'ailleurs pas respectées par les colons), dans la majorité des cas, ce ne sont pas les territoires dans leur ensemble qui sont reconnus comme propriétés indigènes, mais les villages nucléarisés et les terrains cultivés adjacents. Ainsi les communautés natives qui sont reconnues, puis titularisées, se voient attribuer des superficies de terrain dérisoires au regard de leur mode de vie itinérant. Cette situation laisse présager une condamnation à terme de leurs activités traditionnelles.

Dans cette perspective, la situation des habitants de Tayakome et Yomybato s'avère particulière et paradoxale. Certes ils n'ont pas le droit d'exploiter les ressources naturelles de leur territoire à des fins commerciales, alors que l'argent gagné leur permettrait d'accéder à de meilleures conditions sanitaires et éducatives et d'acquérir les biens « industriels » qu'ils valorisent tant. Certes ils doivent respecter, dans une certaine mesure, les contraintes que leur impose l'administration du parc national du Manu. Mais, finalement, jusqu'à présent, vivre au sein de ce même parc national leur donne ce double avantage (non négligeable) d'être protégés de la colonisation de leurs terres et, par la même, de toujours disposer de ressources naturelles relativement abondantes. Par ailleurs, force est de constater que les relations entre les populations matsiguenga de Tayakome et Yomybato et l'administration du parc national du Manu ne sont visiblement plus aussi envenimées qu'auparavant. Obtenir leurs titres de propriété pour commercialiser les ressources de leurs terres n'est plus une priorité pour les Matsiguenga depuis que l'administration consentit enfin à ce qu'ils aient une activité économique rémunérée en guise de compensation des restrictions subies. En effet, I'Institut National des Ressources Naturelles (INRENA) attribua en 1995 une concession aux communautés natives de Tayakome et Yomybato pour qu'elles construisent et gèrent une auberge dans la zone réservée à usage touristique du parc national du Manu.

\section{POUR UNE CONCILIATION AUTOUR DE LA CASA MATSIGUENKA?}

Si c'est à partir des années 1980 que l'activité touristique à destination du parc national du Manu commença à s'organiser et à être réglementée, elle ne prit réellement son essor qu'à partir des années 1990. Paradoxalement, ce sont des scientifiques travaillant à la station biologique de Cocha Cashu qui en promurent le développement. Ceux-ci étaient préoccupés par le devenir du parc national du Manu alors menacé par l'insuffisance des subventions publiques qui ne permettaient pas d'en assurer la protection requise. Le tourisme apparut à leurs yeux comme l'opportunité d'obtenir les fonds nécessaires au bon maintien du parc national, de sensibiliser l'opinion internationale à l'importance de 
préserver des écosystèmes comme celui-ci et de générer des emplois stables profitant aussi aux populations locales qui cesseraient ainsi de mettre en péril la faune et la flore du parc (Munn, 1985 : 18-19). Pour Charles Munn, le tourisme devait aussi profiter aux Matsiguenga. En tant que biologiste, il travaillait depuis plusieurs années en collaboration avec des habitants de Tayakome qui lui servaient de guides, d'assistants ou de motoristes lors de ses séjours à Cocha Cashu. Idéaliste et héritier fortuné en quête d'un nouveau lobby touristique, c'est par l'intermédiaire du CEDIA (dont il était l'un des principaux donateurs) qu'il initia le projet de la Casa Matsiguenka : une auberge touristique gérée par et au profit des habitants de Tayakome et Yomybato.

En 1992, l'administration du parc national du Manu étant hostile au projet, c'est sans son autorisation qu'une première Casa Matsiguenka fut construite... et aussitôt détruite ! Le projet était né, l'opiniâtreté des Matsiguenga et de ses instigateurs allait le rendre viable. Les tensions entre le parc et les Matsiguenga avaient atteint à ce moment-là leur summum. Sous I'impulsion du CEDIA, les habitants de Tayakome et Yomybato étaient décidés à ne pas renoncer. L'INRENA capitula et décida de faire sien le projet touristique en écartant le CEDIA. En 1995, I'INRENA attribua donc aux communautés natives de Tayakome et Yomybato une concession de 6 ha, proche de la Cocha Salvador, dans la zone réservée à usage touristique pour qu'ils y construisent et administrent une auberge. Le projet de la Casa Matsiguenka allait dorénavant être pris en charge et développé dans le cadre du projet FANPE12 : un programme de coopération dirigée par la Coopération technique allemande (Deutsche Gesellschaft für Technische Zusammenarbeit-GTZ) qui, en collaboration avec I'INRENA, se proposait de consolider le Système National des Aires Naturelles Protégées par l'État péruvien (SINANPE).

En 1996, les deux communautés natives durent fonder I'« Entreprise multicommunale matsiguenka» pour gérer leur future auberge. Pour assister dans leur démarche des populations « analphabètes, sans certificat de naissance et document d'identité », le projet FANPE mit en place le monitorat de ces populations. Débuté en 1997, il aurait dû se prolonger jusqu'en 2008 ; mais il prit fin en 2003, la GTZ décidant de financer d'autres projets qui lui parurent davantage prioritaires. Ce monitorat comprenait différentes phases : de l'assistance technique pour la construction d'une auberge avec toutes les commodités requises pour l'accueil des touristes à des ateliers d'éducation environnementale dirigés par une ONG péruvienne pour la conservation de l'environnement (I'APECO).

Pour les habitants de Tayakome et Yomybato, le projet de la Casa Matsiguenka devait enfin leur assurer d'obtenir une source de revenus qui leur permettrait de ne plus dépendre d'occasionnelles donations et d'acheter des biens en commun tels que des médicaments, des fournitures scolaires ou de l'essence pour le bateau communal... ainsi que des biens devenus nécessaires à chaque famille depuis le passage de l'Institut Linguistique d'Été, comme des habits, des outils de métal, des moustiquaires, des ustensiles de cuisine, des denrées (sel, huile), des détergents, etc. Selon les responsables du FANPE, bien plus qu'un moyen de capter les fonds nécessaires aux deux communautés pour développer ou améliorer les secteurs de l'éducation, de la santé et de la communication, ce projet devait permettre aux Matsiguenga de se familiariser progressivement à l'économie de marché, à la gestion d'entreprise et plus spécifiquement à l'activité touristique.

Avec l'appui de la GTZ, I'APECO et certains guides d'autres agences touristiques qui travaillent dans la zone, les Matsiguenga ont appris à répondre aux attentes des touristes, c'est-à-dire à préparer les infrastructures de l'auberge pour leur arrivée, à les accueillir

12 FANPE est le sigle de Fomento del Sistema Nacional de Areas Naturales Protegidas por el Estado. 
en n'oubliant pas de revêtir préalablement leur habit traditionnel, à les guider sur le site de leur auberge et sur les chemins touristiques de la zone, ainsi qu'à réaliser quelques démonstrations de leurs activités journalières (tirer à l'arc, jouer de la musique, raconter des mythes, etc.). Au cours de leur visite au Manu, lorsqu'ils séjournent à la Casa Matsiguenka, les touristes ont ainsi l'occasion de rencontrer l'une des tribus « traditionnelles » locales. La situation actuelle laisse augurer que les Matsiguenga qui travaillent là offriront chaque fois davantage de scènes de leur vie quotidienne, un ensemble de gestes chorégraphiés pour répondre à la quête d'exotisme de leurs visiteurs.

Un tel tourisme constitue un vecteur d'acculturation, capable de provoquer des changements radicaux et irréversibles dans les communautés d'accueil. À court terme, le développement de ce type de tourisme permet aux populations indigènes de conserver «leur » culture ou une culture réinventée, tout en leur fournissant un revenu — dans la mesure où elles récoltent effectivement le fruit de leur travail. Mais comment ce tourisme peut-il être « durable » si I'« objet de consommation » qu'est la « culture primitive » est abandonné sous l'effet d'un pouvoir d'achat croissant de ces derniers représentants ? Dans le cas de la Casa Matsiguenka, celle-ci s'annonçant comme une sorte de vitrine de la culture matsiguenga, elle pourrait permettre dans une moindre mesure de valoriser cette dernière et, en quelque sorte, la préserver puisque présente ou du moins représentée en ce lieu « conservatoire ». Mais une telle «muséification» est-elle souhaitable? N'est-il pas préférable que les populations indigènes impliquées dans un tel projet touristique déterminent elles-mêmes comment gérer leur entreprise et cessent d'être instrumentalisées au profit de personnes extérieures? Sur ce point, je rejoins Aurélie Volle pour qui l'important n'est pas tant de savoir quels sont les impacts (positifs ou négatifs) du tourisme sur les populations indigènes, mais de savoir ce que ces mêmes populations pensent et font du tourisme (Volle, 2005 : 151-152). Ainsi, selon Aurélie Volle

«le tourisme (...), parce qu'il porte le projet de réduire la dépendance et la marginalisation économique et sociale, apparaît donc davantage comme un effort de restructuration et d'adaptation aux conditions contemporaines » (Volle, 2005 : 151-152).

Et elle poursuit en conclusion :

« poser le problème en termes de répartition des pouvoirs est plus éclairant. En effet, le tourisme peut être un outil de contrôle étatique comme il peut être un outil de développement local, si le contrôle culturel demeure entre les mains des intéressés » (Volle, 2005 : 217).

En l'occurrence, depuis la mise en place du projet de la Casa Matsiguenka, loin d'avoir la possibilité de gérer leur auberge comme elles l'entendent, les communautés natives de Tayakome et Yomybato doivent se plier aux décisions prises par l'administration du parc national et le projet FANPE, qui se considèrent comme étant les seuls aptes à déterminer ce qui peut être fait ou non au sein de l'auberge.

\section{UN « DÉVELOPPEMENT » SOUS CONTRÔLE DES AUTORITÉS}

À titre d'exemple, je propose de revenir au fait relaté en introduction. L'administration du parc national du Manu, suivant les conseils judicieux des scientifiques les plus conservateurs, interdit strictement aux Matsiguenga travaillant à l'auberge de cultiver un jardin pour s'alimenter. Le principal argument invoqué par l'administration est l'impact négatif qu'aurait l'ouverture de cet essart sur la faune et la flore de la zone réservée à usage touristique. Elle prétexte également que les touristes visitant le Manu ne viennent pas y voir 
des jardins, mais de la forêt vierge ! D'une part, c'est faire abstraction d'études récentes qui montrent que le bassin amazonien est en partie anthropisé et surtout comment les peuples indigènes ont contribué à l'enrichissement de sa biodiversité, et non à sa détérioration, en I'occurrence par la pratique d'une horticulture itinérante (Balée, 1993). D'autre part, c'est jouer sur l'imaginaire des touristes dont la majorité ignore que, jusqu'à la fin des années 1960, la zone qu'ils visitent était exploitée par des bûcherons en quête de bois précieux et des chasseurs à la recherche de peaux de loutres, jaguars, caïmans, etc. Outre de renseigner sur l'idéologie conservationniste qui préside la politique du parc, cet exemple illustre également les contradictions existantes dans la gestion du parc vis-à-vis des populations matsiguenga.

L'auberge étant installée loin des communautés, cette interdiction de cultiver un jardin oblige les Matsiguenga à consommer des produits achetés à l'extérieur, plus facilement acheminables par les bateaux touristiques. Paradoxalement, si le panneau informatif qui se trouve à l'entrée du parc national du Manu, au poste de contrôle de Acjanaco, indique que parmi les objectifs du parc, l'un d'eux est de " préserver le patrimoine culturel des Populations Indigènes de l'Aire », I'administration du parc impose finalement aux dites populations de changer leur diète alimentaire et de se nourrir de pâtes, riz, thon et sardines en boîte, etc. C'est ainsi que le projet de la Casa Matsiguenka, qui s'était élaboré avec l'objectif de «minimiser les impacts négatifs sur la culture des populations indigènes participantes », les a pourtant affectées en modifiant l'une des principales composantes de leur identité culturelle : leur alimentation. Est-il nécessaire de rappeler, à la suite de Claude Fischler (1993 : 66-70), que tant sur le plan biologique et nutritionnel que sur le plan social et imaginaire, nous sommes ce que nous mangeons ?

Pour les Matsiguenga, il n'est pas anodin de se voir interdire l'ouverture d'un jardin. Les produits horticoles sont à la base de leur alimentation traditionnelle et leur fournissent 80 à $90 \%$ des apports nutritifs quotidiens. Le manioc doux (Manihot esculenta) leur en apporte à lui seul 70 à $80 \%$ (Ohl, 2004 : 16). L'étymologie matsiguenga elle-même souligne l'importance du manioc : effectivement si celui-ci se dit en matsiguenga sekatsi, sekatagantsi signifie «manger». Le manioc est donc bien l'aliment de base par excellence. Et ce n'est pas non plus fortuit si le premier mythe que Don Cornelio choisit de me raconter fut celui où Kashiri, "Monsieur Lune», enseigna aux Anciens (qui se nourrissaient alors de boue) comment cultiver le manioc et les autres plantes horticoles. En être privé peut avoir de graves conséquences à plus ou moins long terme. Comme il l'a été observé dans $d^{\prime}$ 'autres cas ${ }^{13}$, un tel changement dans la diète alimentaire peut entraîner une détérioration de l'état de santé - causant une dénutrition, des maladies du métabolisme ou encore des caries..., en particulier chez les enfants en bas âge. Par ailleurs, les rendre peu à peu dépendants de produits commercialisés peut aussi avoir des répercussions sur le mode de vie des habitants de Tayakome et Yomybato qui consacrent aujourd'hui encore la majorité de leurs activités socio-économiques à la production de leurs moyens de subsistance en quasi totale autonomie.

Depuis que le projet fut initié, les Matsiguenga travaillant à l'auberge demandent régulièrement aux autorités leur accord pour ouvrir un essart afin d'assurer leur alimentation. Face au refus constant de celles-ci, ils prirent tout de même l'initiative de mettre une parcelle en culture un peu à l'écart de l'auberge. Nous avons vu que la réponse des autorités ne se fit pas attendre : une fois le jardin découvert, celui-ci fut mis à sac par les gardes du parc

13 Sont entre autres cités par Julia Ohl (2004: 33) : Bennett, B., 1996 - La Traida y el Destierro de Inaenka. In : Globalización y Cambio en la Amazonía Indigena (Santos Granero, F., ed.) : 341-367 ; Quito : Abya-Yala ; ou encore : Fleuret, P. \& Fleuret, A., 1980 - Nutrition, Consumption and Agricultural Change. Human Organization, 39: 250-260. 
national. Estimant légitime la volonté des habitants de Tayakome et Yomybato, la docteur en biologie Julia Ohl et moi-même avons présenté le 27 mai 2004 une requête auprès de I'Intendant Général des Aires Naturelles Protégées alors en poste, afin que soit autorisée I'ouverture d'un essart aux abords de la Casa Matsiguenka. Si notre sollicitation avait reçue un avis favorable, aux dernières nouvelles aucune autorisation légale n'a été émise en ce sens. Pourtant, selon les estimations de Julia Ohl, environ 0,385 ha suffiraient chaque année à assurer l'alimentation des dix personnes travaillant à l'auberge. En prenant en compte la rotation annuelle pratiquée ainsi que la mise en jachère sur une période de 20 ans, au total 7,7 ha sont nécessaires pour garantir une production horticole viable. Que sont 7,7 ha sur les 1716 295,48 ha que comprend le parc national dans son extension la plus récente (redéfinie par Décret Suprême le 14 juillet 2002)14 ? Pour ainsi dire une goutte d'eau dans un océan, mais tout un symbole pour les autorités, garantes de l'intégrité du parc national du Manu.

\section{PARCS NATURELS, DIALECTIQUES CULTURELLES}

Depuis une cinquantaine d'années, ayant constaté l'épuisement de certaines ressources naturelles, minières, végétales ou animales, et l'incapacité, à l'échelle planétaire, de maîtriser la croissance démographique qui menace les équilibres et les cycles de renouvellement des ressources restantes, de plus en plus d'acteurs considèrent la conservation de la Nature comme un enjeu majeur pour le devenir de l'Humanité et de la Terre en général. Cette conservation est en particulier assurée via l'établissement d'aires naturelles protégées — qui sont autant d'échantillons représentatifs des divers écosystèmes rencontrés à travers le monde. Il est cependant important de rappeler que c'est dans une toute autre perspective que le premier parc national fut créé en 1872 aux États-Unis. La création du parc national du Yellowstone fut l'une des premières décisions politiques en faveur de la conservation de la nature, prise alors par le Président Ulysse Grant.

D'après Michel Conan (1985 : 179-180), au milieu du XIXe siècle, I'Amérique se démarque de la civilisation européenne et de son goût prononcé pour les paysages cultivés. Pendant la Conquête de l'Ouest, la découverte de grands espaces « sauvages » remplit très vite une fonction morale. Ces espaces, où Dieu se manifeste directement aux hommes, lavés des souillures urbaines et des faux semblants de I'Ancien Monde, contribuent à la formation d'un homme véritablement américain. Dès l'origine des parcs nationaux, ces espaces naturels représentent donc une ressource qui ne doit pas être exploitée par l'homme mais gardée intacte. Cette conception porte en elle le germe de la radicalisation des mouvements écologistes telle qu'on l'observe depuis les années 1960. En effet, pour les défenseurs de la deep ecology, la Nature, élevée au rang d'un absolu en soi, doit être protégée de l'Homme en général, un Homme qui lui est nuisible par essence. Selon ce précepte et suivant le modèle nord-américain, pendant des décennies, I'instauration de parcs nationaux s'accompagna de I'expulsion massive des populations autochtones. Ce fut en particulier le cas en Afrique, où, au nom de la conservation, un million de kilomètres carrés de terres cultivées, de pâtures et de forêts ont été vidées de leurs habitants au cours du siècle dernier (Colchester, 2001 : 12). Bien que les études chiffrées fassent défaut, on estime que plusieurs millions de personnes ont été ainsi déplacées (Colchester, 2001 : 12). Ces délocalisations, qui se multiplièrent il y a une quarantaine d'années, se poursuivent aujourd'hui. Par exemple au Botswana, en 1997 puis de nouveau en 2002, plusieurs milliers de San (Bushmen) ont été expulsés de leurs terres, localisées à l'intérieur de la réserve naturelle du Kalahari central qui avait pourtant été

14 D'après le Décret Suprême N 045-2002-AG (INRENA-SPMA, 2002 : 198-199). 
créée à leur intention en 196115. Pour satisfaire les visées conservationnistes, les territoires des populations autochtones devraient idéalement être transformés en espaces intangibles, c'est-à-dire préservés de toute présence humaine. Une telle approche ne revêt aucune signification - si ce n'est qu'elle constitue une nouvelle forme de spoliation territoriale pour les peuples indigènes qui en sont les témoins et, en l'occurrence, les victimes. Pour reprendre Philippe Descola (1999 : 215), « pour que l'on ait pu songer à protéger la nature, il fallait d'abord que la nature existât en tant que telle ».

La «nature» est, pour les Occidentaux, ce qui existe en dehors du monde humanisé, transformé par I'homme. Inséparable du milieu artificiel qu'il s'est créé, l'Occident la perçoit comme une réalité extérieure à la culture, celle-ci étant définie a contrario comme l'ensemble des faits humains. Or, si la pensée occidentale a établi un dualisme entre les concepts de nature et de culture, ce modèle est étranger à de nombreuses populations. Reste-t-il en effet une place pour le concept de nature dans des cosmologies où humains, plantes et animaux partagent une même essence, où les caractéristiques de I'humanité sont attribuées aux «êtres de la nature » et où humains et non-humains entretiennent des relations sociales calquées sur le modèle offert par la société humaine (cf. Arhem, 1996; Chaumeil, 1983 ; Descola, 1986) ? Notre concept de nature est donc à repenser ; du moins sa prétendue universalité doit être relativisée tant dans le cadre théorique de la recherche scientifique que dans la pratique des intervenants dans la préservation ou la gestion des espaces dits « naturels ».

Pour les Matsiguenga, s'il est difficilement compréhensible de vouloir délimiter un espace dont I'homme serait exclu, la raison d'être du parc, conserver la nature, est tout aussi inintelligible. Non seulement parce que la dite «nature » n'a pas d'existence en soi - en tant que sphère autonome de la vie sociale - , mais surtout parce qu'ils considèrent les non-humains comme éternels. Effectivement, d'après Dan Rosengren (1987 : 51), Kipatsi, le troisième monde parmi les cinq mondes de la cosmologie matsiguenga : la Terre, omniprésente et éternelle, est composée de divers éléments (collines, forêts, rivières, etc.), qui, s'ils ne sont pas omniprésents, ne sont pas pour autant dépourvus des caractéristiques temporelles de Kipatsi dont ils sont la continuité. Ils ont toujours été là et seront toujours là, peu importe ce que l'homme désire ou puisse en faire. Si les Matsiguenga de Tayakome et Yomybato pensent en théorie que les « ressources naturelles»sont inépuisables, dans la pratique, ils ont effectivement des jardins toujours aussi fertiles et rencontrent autant de gibier et de poisson qu'autrefois aux alentours de leur communauté. Ainsi, pour les Matsiguenga, Kipatsi se régénère éternellement et il n'est donc nul besoin de la « protéger », ni de la « conserver ».

Cependant cette perspective est en train d'être bouleversée. Confrontés à de nouveaux modes d'exploitation des ressources naturelles, les Matsiguenga ont pu à maintes reprises en évaluer les conséquences. C'est plus particulièrement le cas des populations originaires du bassin de I'Urubamba. Depuis plusieurs générations, celles-ci sont victimes de la colonisation de leurs terres et ont vu prospérer et se succéder nombre d'activités extractives et productives jusqu'à la raréfaction, voire disparition des ressources dont eux-mêmes dépendaient pour vivre. Plus récemment, l'exploitation de gisements de gaz dans la région du Camisea oblige de nombreuses familles à vivre retranchées sur des terres impropres à I'horticulture, où le gibier est absent. Lors d'occasionnelles rencontres, les Matsiguenga, natifs de cette région, témoignent de cette dure réalité auprès de ceux qui habitent dans le Manu et louent les avantages que ces derniers ont à vivre dans un espace ainsi protégé de la présence des colons.

15 D'après Survival International et I'Association Américaine d'Anthropologie. Informations disponibles sur : http:// www.survivalfrance.org/press_room.php?id=1554 http://www.aaanet.org/committees/cfhr/rptbotswana.htm 


\section{EN CONCLUSION}

Pour les Matsiguenga de Tayakome et Yomybato, vivre dans le parc national du Manu présente des aspects positifs et négatifs. Si, selon eux, celui-ci leur confère une protection contre l'invasion de leurs terres et leur permet entre autres de continuer à jouir des ressources qu'ils exploitent couramment, ils ne le considèrent pas moins comme un frein à leur développement. D'après Michael Baksh, la qualité de vie des Matsiguenga est à présent étroitement liée à leurs capacités à générer de l'argent (1995 : 190-191), — du moins à acquérir des biens industriels, faudrait-il préciser. Car nombreux sont les Matsiguenga qui n'ont encore aucun contact direct avec la société marchande et arrivent cependant à se procurer les objets qu'ils désirent en faisant du troc. Dans le Manu par exemple, les familles qui vivent « isolées » sur les hauts Cumerjali et Sotileja, visitent chaque année, au moment de l'étiage, les habitants de Yomybato et en profitent pour échanger avec eux des produits sylvestres : escargots, fruits, graines, résine, etc., contre les biens si prisés : habits, haches, machettes, couteaux, casseroles, etc., - excepté lorsque les habitants de Yomybato manquent eux-mêmes de ces objets et se trouvent par conséquent dans l'impossibilité de les fournir à leurs pairs. De leur côté, les habitants de Tayakome et Yomybato bénéficient généralement de donations provenant de divers groupes d'intérêts : missionnaires, ONG, scientifiques, Ministère de la Santé, Ministère de l'Éducation, etc. Mais d'une part ces donations ne leur parviennent pas de façon constante : c'est par exemple ainsi que, plusieurs mois par an, les dispensaires, installés dans chacune des deux communautés depuis 1996, n'ont plus suffisamment de médicament en réserve, ou que les élèves s'évertuent à poursuivre leur scolarité sans cahier, ni stylo. Et ce, bien que les secteurs de la santé et de l'éducation reçoivent un important soutien des organisations religieuses caritatives, des ONG et de l'État qui en font des objectifs prioritaires de leurs programmes de développement. D'autre part, les donations reçues par les habitants de Tayakome et Yomybato ne couvrent qu'une partie de leurs besoins. Car aux biens jugés utiles par les donateurs comme les moustiquaires, les médicaments, les fournitures scolaires, les habits, les outils de métal, etc., s'ajoutent des biens prestigieux pour les Matsiguenga, tels que les montres, les parfums ou encore les chaînes hi-fi à la mode ces temps-ci pour organiser au mieux les fêtes de bière de manioc sur fond de musique huayno ou cumbia. Pour acquérir ces nouveaux objets, il est certain qu'avoir des revenus devient nécessaire.

En ce sens, l'interdiction faite d'utiliser les ressources naturelles à des fins commerciales à l'intérieur du parc national apparaît comme particulièrement contraignante pour les habitants de Tayakome et Yomybato et oblige les hommes à partir à l'extérieur vendre ou échanger leur force de travail afin de pouvoir satisfaire leurs besoins et ceux de leur famille. Ainsi, pendant des années, les habitants de Tayakome et Yomybato voulurent obtenir les titres de propriété de leurs terres davantage pour être libre d'en exploiter les ressources comme ils le souhaitaient que pour se prémunir de la colonisation. Encore aurait-il fallu trouver des activités économiquement rentables : étant donné l'éloignement de ces deux communautés natives des principaux centres de marchés locaux, les coûts de transports sont trop élevés et les prix de vente de la plupart des marchandises trop bas (Ohl, 2004 : 21). Si toutes les chances étaient mises du côté des habitants de Tayakome et Yomybato pour qu'ils puissent mener à bien le projet de la Casa Matsiguenka, le tourisme serait assurément la meilleure opportunité du moment. Mais il ne s'agit pas ici de savoir quelles alternatives économiques pourraient profiter aux habitants de Tayakome et Yomybato mais bien de s'interroger sur les possibilités que ceux-ci ont actuellement d'exercer sur leurs terres les activités de leur choix. En d'autres termes, la principale question soulevée par cet article consacré aux interrelations entre populations matsiguenga et administration 
du parc national du Manu est celle de la légitimité que les uns et les autres ont sur ce territoire partagé.

En l'occurrence, comme nous avons pu le constater, depuis que le parc national du Manu a été instauré, l'autorité et la légitimité des habitants de Tayakome et Yomybato semblent bien réduites en comparaison du caractère hégémonique de cette organisation venue s'imposer à eux. En effet, lorsque le bassin du Manu fut reconnu parc national, I'administration - pour établir sa souveraineté sur ce territoire qui lui fut concédé par décret légal — dut non seulement employer force armée et dissuasion, mais aussi instaurer un ensemble de normes réglementant les modalités, dorénavant seules autorisées, d'accès et d'usage de cet espace. Peu importe alors que le dit espace fût déjà habité et que ses habitants y aient des modes d'appropriation territoriale et d'exploitation des ressources différents. Si les populations indigènes du Manu furent finalement tolérées à l'intérieur de cette aire naturelle protégée, aujourd'hui encore elles n'en sont pas moins perçues par les conservationnistes comme des intrus dont les activités socio-économiques sont nuisibles à l'intégrité de la Nature et par conséquent à celle du parc national. Ainsi, bien que les institutions chargées de la conservation tendent ces dernières années à appliquer de nouvelles stratégies, en apparence moins contraignantes et plus conciliantes, un parc national comme celui du Manu demeure, pour la majorité des personnes chargées d'en assurer le maintien, une zone de non droit pour les populations locales, où seuls les intérêts de la conservation prévalent. Et si, du contrôle manu militari à la concession de la Casa Matsiguenka, les configurations de l'interface entre l'administration du parc national du Manu et les Matsiguenga de Tayakome et Yomybato ont manifestement changé, nous avons vu que ces derniers n'en demeurent pas moins contraints de se conformer aux décisions qui leur sont imposées, sans que soit respectée, ni même prise en considération, leur propre volonté. L'interdiction de cultiver un jardin à proximité de leur auberge en est un exemple édifiant. Au regard de la situation actuelle, rien ne laisse présager que les Matsiguenga du Manu pourront un jour décider librement de leur avenir. Pourtant, en juillet 1996, le Pérou compta parmi les pays qui ratifièrent la Convention 169 relative aux peuples indigènes et tribaux, adoptée en 1989 par l'Organisation Internationale du Travail (OIT). Qu'est-il advenu de l'application de cette convention, fondée sur le respect des cultures des peuples indigènes et sur leurs droits à une existence et à un développement continus axés sur les orientations de leur choix?

Dans la seconde partie de la Convention 169, intitulée « Terres », I'OIT insiste sur le fait que «les droits de propriété et de possession sur les terres qu'ils occupent traditionnellement doivent être reconnus aux peuples intéressés» (art. 14). Effectivement, pour l'ensemble des peuples indigènes, en Amazonie comme ailleurs, il est crucial que leur territoire soit reconnu comme tel, condition sine qua non pour assurer tant les conditions matérielles de leur existence que leur reproduction sociale et culturelle de manière autonome. Le terme « reproduction » ne semble d'ailleurs pas ici si approprié tant l'enjeu n'est pas pour ces peuples de se reproduire «à l'identique », mais de déterminer librement leur devenir. Aujourd'hui, alors que la majorité des peuples indigènes sont confrontés à la colonisation et doivent composer avec les États dont les territoires nationaux incluent leurs propres territoires, la libre autodétermination requise sur leurs terres leur est rarement concédée. II apparaît que l'État, selon son modèle centralisateur actuel, peut difficilement reconnaître des collectivités territoriales autonomes. D'ailleurs, serait-il cohérent pour l'État d'attribuer leur indépendance à certains alors qu'il est par définition le garant du Bien commun de l'ensemble de ses concitoyens ? Dans le cas qui nous intéresse ici, la Constitution péruvienne reconnaissant à tout individu le droit «à la vie, à son identité, à son intégrité morale, psychique et physique et à son libre développement et bien-être », n'est-il pas envisageable que les peuples indigènes, vivant actuellement au Pérou, jouissent également de ces droits 
fondamentaux inhérents à la personne ? En sachant et reconnaissant, aujourd'hui plus que jamais, combien il est important pour la continuité de ces peuples que les titres de propriété de leurs terres leur soient attribués, l'État ne devrait-il pas effectivement leur permettre de disposer de leur territoire dans sa totalité et en parfaite autonomie? Comme nous I'avons mentionné (cf. supra), si les deux lois promulguées dans les années 1970 en faveur des communautés natives marquèrent une première étape vers la reconnaissance légale des populations de l'Amazonie péruvienne, le concept même de «communauté native » ne correspond guère aux relations que ces populations entretiennent avec leurs territoires. Généralement semi itinérantes et dispersées sur de vastes territoires, les populations amazoniennes, qui se voient attribuer des terres dont la superficie est exiguë, se trouvent alors dans l'obligation de se sédentariser et donc de changer leurs modes d'exploitation des ressources naturelles, impliquant de leur part qu'elles s'intègrent chaque fois davantage à la société marchande afin de compenser les besoins auxquels elles ne peuvent plus subvenir elles-mêmes. Néanmoins, la promulgation en mai dernier d'une loi « pour la protection des peuples indigènes en situation d'isolement et en situation de contact initial » constitue une nouvelle avancée sur le plan juridique en faveur des peuples indigènes : l'État y garantit, entre autres, de

" protéger leur culture et leurs modes traditionnels de vie, reconnaissant la relation spirituelle particulière de ces peuples avec leur habitat, comme élément constitutif de leur identité » et $d^{\prime}$ « établir des réserves indigènes, déterminées sur la base des aires qu'ils occupent ou auxquelles ils ont accès traditionnellement, jusqu'à ce qu'il décident de l'attribution de titres de propriété de façon volontaire ».

Cependant il est regrettable que le caractère intangible des dites réserves indigènes ne soit que «transitoire », le temps que ces peuples maintiennent leur isolement. Outre leur imprescriptibilité, l'inaliénabilité de ces réserves n'est pas non plus reconnue, puisque cette loi stipule que seuls les peuples indigènes qui les habitent auront le droit d'accéder librement à ces réserves, de s'y établir ou d'en exploiter les ressources naturelles, excepté «au cas où $s^{\prime} y$ trouve une ressource naturelle susceptible d'utilisation et dont l'exploitation résulte de nécessité publique pour l'État (...)». Lorsque l'on sait la connivence qui existe entre les dirigeants des pays « en voie de développement » et les grandes firmes internationales pétrolières, gazières, minières ou autres 16 , on ne peut que se demander dans quelles mesures cette fois-ci les droits des peuples indigènes seront respectés. Reste à savoir si la raison d'État se doit d'être économique ou éthique?

\section{Références citées}

ANS, A.-M. (d'), 1970 - Les tribus indigènes du Parc National du Manu. In : Actas del XXXIX Congreso Internacional de Americanistas, vol. 4 : 95-100 ; Lima.

ANS, A.-M. (d'), 1981 - Encounter in Peru. In : Is God an American? An Anthropological Perspective on the Missionary Work of the Summer Institute of Linguistics (S. Hvalkof \& P. Aaby, eds.) : 145-162 ; Copenhague : IWGIA, Survival international.

ÅRHEM, K., 1996 - The cosmic food web. Human-nature relatedness in the Northwest Amazon. In : Nature and society (P. Descola \& G. Palsson, eds.) : 185-204 ; London : Routledge. 
BAKSH, M., 1995 - Changes in Machiguenga quality of life. In : Indigenous peoples and the future of Amazonia. An ecological Anthropology of an Endangered World (L. E. Sponsel, ed.) : 187-205; Tucson and London : The University of Arizona Press.

BALEE, W., 1993 - Indigenous transformation of Amazonian Forests. An exemple from Marahão, Brazil. L'Homme, 126-128, XXXIII (2-4) : 231-254. Numéro spécial: La remontée de l'Amazone. Anthropologie et histoire des sociétés amazoniennes.

BOUKHARI, S., 1999 - Les droits autochtones contre la mainmise des entreprises. Courrier de I'Unesco (avril) : 10-11.

CHAUMEIL, J.-P., 1983 - Voir, savoir, pouvoir : le chamanisme chez les Yagua du Nord-est péruvien, 352 p. ; Paris : EHESS.

COLCHESTER, M., 1999 - Parcs ou peuples ? In : Nature sauvage, nature sauvée ? Ecologie et peuples autochtones (J.-C. Monod \& J.-P. Razon, eds.) : 159-193 ; Paris : Peuples autochtones et développement. Ethnies-documents 24-25.

COLCHESTER, M., 2001 - Peut-on préserver la nature au détriment des hommes ? Le Courrier de l'UNESCO (juillet-août) : 12-15.

CONAN, M., 1985 - Découverte et invention du Yellowstone. Esquisse de I'histoire de la création d'une culture visuelle aux États-Unis au $19^{\text {e }}$ siècle. In : Protection de la nature. Histoire et idéologie. De la nature à l'environnement (A. Cadoret, ed.) : 175192 ; Paris : I'Harmattan.

DESCOLA, P., 1986 - La Nature domestique. Symbolique et praxis dans l'écologie des Achuar, 450 p. ; Paris : Maison des Sciences de l'Homme.

DESCOLA, P., 1999 - Diversité biologique, diversité culturelle. In : Nature sauvage, nature sauvée ? Écologie et peuples autochtones (J.-C. Monod \& J.-P. Razon, eds.) : 213235 ; Paris : Peuples autochtones et développement. Ethnies-documents 24-25.

FISCHLER, C., 1993 - L'Homnivore, 446 p. ; Paris : Odile Jacob.

INRENA-SPMA, 2002 - Compendio de legislación de Áreas Naturales Protegidas, 493 p. ; Lima.

MUNN, C., 1985 - Ciencia y turismo en la Reserva de la Biosfera del Manu. Boletín de Lima, 42 : 9-26 ; Lima.

OHL, J., 2004 - iEl eco-turismo como oportunidad para un desarrollo sostenible? La economía de los Matsiguenkas en el Parque Nacional del Manu, 110 p. ; Eschborn : GTZ-TOEB.

RENARD-CASEVITZ, F.-M., 1972 - Les Matsiguenga. Journal de la Société des Américanistes, LXI : 215-253.

ROSENGREN, D., 1987 - Concepciones de trabajo y relaciones sociales en el uso de la tierra entre los Matsiguenka del Alto Urubamba. Amazonia peruana, 8 (14) : 39-59.

RUMMENHOELLER, K. \& HELBERG CHAVEZ, H., 1992 - Decisiones ecológicas al margen de los indios: El Parque Nacional del Manu (Peru). América indígena, 52 (4) : 249-266.

VITERI GUALINGA, C., 1999 - Le pétrole coule, la forêt saigne. Courrier de I'Unesco (avril) : 12-13.

VOLLE, A., 2005 - Quand les Mapuche optent pour le tourisme. Regards croisés sur le développement au Chili, 227 p. ; Paris : L'Harmattan (collection Tourismes et sociétés). 